\title{
Microsymposium
}

MS060.002

\section{RIXS at extreme conditions at the GALAXIES beamline}

\author{
Jean-Pascal Rueff ${ }^{1}$ \\ ${ }^{1}$ Synchrotron SOLEIL, Gif Sur Yvette, France \\ E-mail: jean-pascal.rueff@synchrotron-soleil.fr
}

RIXS is a well-established probe of the electronic and magnetic properties of materials. As an all photon technique, RIXS in the hard $x$-ray range is well suited to investigate materials at extreme conditions thanks to its large penetration depth and its chemical and orbital sensitivity. In this presentation, we will focus on recent results obtained by RIXS on the newly commissioned GALAXIES beamline at SOLEIL synchrotron [1]. We will more specifically address magnetic and electronic changes occurring at high pressure and low temperature in the Fe-based superconductors [2,3] and correlated oxides. Perspectives for RIXS imaging at high pressure will be discussed.

[1] Rueff J.-P. et al. (2015), J. Synchrotron Rad. 22, 175

[2] Balédent V. et al. (2015), Phys. Rev. Lett. 114, 177001.

[3] Lebert B. et al. (2017), in prep.

Keywords: RIXS, high pressure, superconductors 